\title{
A third-order compact nonlinear scheme for compressible flow simulations
}

\author{
Zhuangzhuang Tian ${ }^{\mathrm{a}}$, Guangxue Wang ${ }^{\mathrm{a}}$, Fan Zhang, ${ }^{\mathrm{b}}$, Huaibao Zhanga,* \\ ${ }^{a}$ School of Physics, Sun Yat-sen University, Guangzhou, Guangdong 510006, China \\ ${ }^{b}$ Centre for mathematical Plasma-Astrophysics, Department of Mathematics, KU Leuven, Celestijnenlaan 200 B, 3001 \\ Leuven, Belgium
}

\begin{abstract}
Reynolds-averaged Navier-Stokes (RANS) simulations based on second-order numerical methods are widely used by commercial codes, and work as dominating tools for most industrial applications. They, however, suffer from limitations in accurate and reliable predictions of skin-friction drag and aerodynamic heating, as well as in simulations of complex flows such as large-scale separation and transition. A remedy for this is the development of high-order schemes, by which numerically-induced dissipation and dispersion errors of low-order schemes can be effectively reduced. Weighted compact nonlinear schemes (WCNSs) [1] are a family of high resolution nonlinear shock-capturing methods. A stencil-selection procedure is introduced in the proposed work with an aim to improve the nonlinear weight of the third-order WCNS. By using the approximate dispersion relation (ADR) analysis, it is demonstrated that the new scheme has reduced the dissipation and dispersion errors, compared with WCNSs using two typical nonlinear weights. Improvements are also achieved by the new scheme in numerical tests such as the double Mach reflection problem and the Rayleigh-Taylor instability simulation, which are characterized by strong shock discontinuities and rich small scales, respectively. The new scheme is therefore highly favored in the simulation of flow problems involving strong discontinuities and multi-scales phenomena.

Keywords: third-order; compact nonlinear scheme; nonlinear interpolation; ENO; compressible flows
\end{abstract}

\section{Introduction}

Accurate predictions of a compressible flow field involving discontinuities such as shocks and interfaces, as well as small-scale flow structures, have received considerable attention in the computational fluid dynamics (CFD) research community over the past decades. Second-order accurate RANS solvers are no doubt the

(or higher) order accurate in smooth regions may yield oscillations wherever the solution is discontinuous. Such kind of oscillation, also called Gibbs phenomenon, does not decay in magnitude as the mesh is refined [3, 4]. In order to address the issue, Harten [5] introduced the concept of total-variation diminishing (TVD) schemes. Under this concept, a measure of discrete variation in the solution field is defined, and it is required

\footnotetext{
*Corresponding author.

Email addresses: fan.zhang@kuleuven.be (Fan Zhang), zhanghb28@mail.sysu.edu.cn, huaibao.zhang@uky.edu (Huaibao Zhang)
} 
to decrease during the time evolution in order for the scheme developed to prevent the generation of new extrema in the flow solution.

Whereas in past few decades second-order accurate methods with TVD limiters have been widely used for engineering applications mostly due to their robustness and efficiency in obtaining decent solutions, they have limitations in accurate and reliable computations of specific problems, for instance, skin-friction drag and aerodynamic heating prediction. The main reason lies in the fact that variables - aerodynamic heat transfer rate to the wall surface for instance - is a function of temperature gradient, for which only first-order accurate solutions can be obtained by a second-order accurate method in the boundary layer. Additionally, predictions of aforementioned variables are also found highly sensitive to local grid density and geometrical mesh quality near the surface as a second-order method is used. More inherent limitations of second-order methods can be clearly seen when it comes to the simulations of turbulence flows, such as vortical flow, large-scale separation and transition. High-order schemes (third order accurate or higher) are invoked to overcome difficulties encountered by low-order schemes. Numerically-induced dissipation and dispersion errors of low-order schemes can be effectively reduced [6]. It has also been recognized that high efficiency is achieved by high-order schemes over low-order schemes in CPU time when obtaining the same level of accuracy for a solution since a coarser grid is sufficient 2. Additionally, high-order schemes are promising since they offer superior parallel scalability and efficiency compared against low-order schemes for the same meshes [7, 8.

Among high-order shock-capturing schemes, a popular family is the weighted compact nonlinear schemes (WCNSs) developed by Deng and Zhang 11. WCNS is an extension of the compact nonlinear scheme compact schemes proposed by Lele [6], among others. Past research has been performed on WCNS schemes, notably by Nonomura et al. [10, 11, Deng et al. [12, 13, and Wong and Lele 14, showing that WCNSs have several advantages over the standard finite-difference WENO schemes of Liu et al. [15]: (1) resolution is slightly higher; (2) choice of flux schemes is more flexible - see, e.g., Roe [16, van Leer [17], and Wang et al. [18, respectively; and (3) WCNSs perform well with respect to freestream and vortex preservation properties on wavy grids [10. Of course, it is worth noted that the WENO schemes are more robust than the WCNSs, and thus specific effort needs to be made to improve the robustness of WCNSs [11.

The classical WCNS procedure consists of three steps [1: (1) node-to-midpoint weighted nonlinear interpolation of flow variables, (2) evaluation of fluxes at midpoints, and (3) midpoint-to-node centered flux differencing. In the first step the idea of nonlinear weighting, originating from WENO schemes [15, is used. The classical WCNS using the JS weight of Jiang and Shu [19] is therefore referred to as WCNS-JS. It has been demonstrated that the nonlinear interpolation procedure is essential for the shock capturing ability and resolution of WCNSs [20, 21, 22. Hence, improving this procedure has been attracting much attention.

In the proposed work, we aim to develop a high-resolution third-order compact nonlinear scheme, since it ${ }_{45}$ is simple and efficient in practical applications. Recently, we incorporated the stencil-selection procedure 23 , 
24] to improve WCNS, resulting fifth-order and sixth-order low-dissipation compact nonlinear scheme [25, 26] within the framework of WCNS, exhibiting superior spectral properties and shock-capturing capabilities. The same concept of stencil-selection is applied in this work, yet a more efficient nonlinear function, which is built in the form of the JS weight [19, is adopted for the present scheme to measure the smoothness of candidate stencils. For introducing the ideas of this work, the remainder of this article is organized as follows. In the next section, the fundamentals and basic equations of compact nonlinear schemes are introduced. Section 3 presents solutions of a scalar equation and the Euler equations, and concluding remarks are given in the last section.

\section{Fundamentals}

The governing equations of compressible flow are hyperbolic systems. Without loss of generality, the one-dimensional scalar hyperbolic conservation law can be first used to discuss numerical schemes involved in this work; this takes the form

$$
\frac{\partial u}{\partial t}+\frac{\partial f(u)}{\partial x}=0
$$

subject to the initial condition

$$
u(x, 0)=u_{0}(x),
$$

where $u$ is the dependent variable, and $f(u)$ is the flux term. The governing equation Eq.(1), together with its initial condition Eq. (2), composes an initial-value problem (IVP), which is assumed to be well-posed in the sense that the solution $u$ continuously depends on the initial field; and the solution is piecewise smooth with a finite number of discontinuities.

Spatial discretization of Eq. (1) is performed on an equally-spaced grid in one dimension with distance between two adjacent grid nodes denoted as $h$. At each node $i, x_{i}=i h$, and $u_{i}=u\left(x_{i}, t\right)$. The characteristic velocity in Eq. (1) is $\partial f(u) / \partial u$, which is assumed to be positive, without loss of generality. Therefore, at each node, a semi-discrete solution can be constructed:

$$
\left(\frac{\mathrm{d} u}{\mathrm{~d} t}\right)_{i}=-\hat{f}_{i}^{\prime}, \quad i=1, \cdots, n
$$

where $\hat{f}_{i}^{\prime}$ is the approximation to the first spatial derivative of the numerical flux function $f_{i}$.

In order to maintain strong robustness, the fourth-order explicit formula provided in [11, i.e., the fourthorder MND, is specifically applied in this work. The formula is given by

$$
\hat{f}_{i}^{\prime}=\frac{b_{1}}{h}\left(\hat{f}_{i+\frac{1}{2}}-\hat{f}_{i-\frac{1}{2}}\right)+\frac{b_{2}}{h}\left(f_{i+1}-f_{i-1}\right),
$$

60 a compact scheme but is considered a special case of general compact nonlinear schemes [12].

Midpoint flux terms in Eq. (4) are unknown and can be evaluated using numerical upwind flux functions. The scalar upwind flux function is given in generic form as

$$
\hat{f}_{i \pm \frac{1}{2}}=\frac{1}{2}\left[\left(f\left(u_{R, i \pm \frac{1}{2}}\right)+f\left(u_{L, i \pm \frac{1}{2}}\right)\right)-|\hat{a}|\left(u_{R, i \pm \frac{1}{2}}-u_{L, i \pm \frac{1}{2}}\right)\right],
$$


where the subscripts, viz., $L$ and $R$, indicate, respectively, the variables on the left- and right-hand sides of the midpoint $x_{i \pm \frac{1}{2}}$; and $\hat{a}$ is the approximate eigenvalue of the flux Jacobian matrix. The method for calculating the midpoint variables $u_{L / R, i \pm \frac{1}{2}}$ is described in the next section.

Once the numerical approximation $\hat{f}_{i}^{\prime}$ is given, temporal intgration of Eq. (3) is performed using the third-order strongly stable Runge-Kutta method [27].

\subsection{Node-to-midpoint interpolation}

As mentioned above, in order to calculate $u_{L / R, i \pm \frac{1}{2}}$, we proposed an improved fifth-order compact nonlinear scheme [25] using a stencil-selection procedure [23] in the nonlinear interpolation. This is carried out by first introducing a smoothness measurement technique followed by a cut-off procedure.

For simplicity, we only consider the evaluation of variables on the left-hand side of $x_{i+\frac{1}{2}}$, i.e., $u_{L, i+\frac{1}{2}}$. The interpolations of $u_{R, i+\frac{1}{2}}$ are performed by using a symmetrical form of $u_{L, i+\frac{1}{2}}$. The subscript $L$ is further dropped for simplicity in the discussions of the following work.

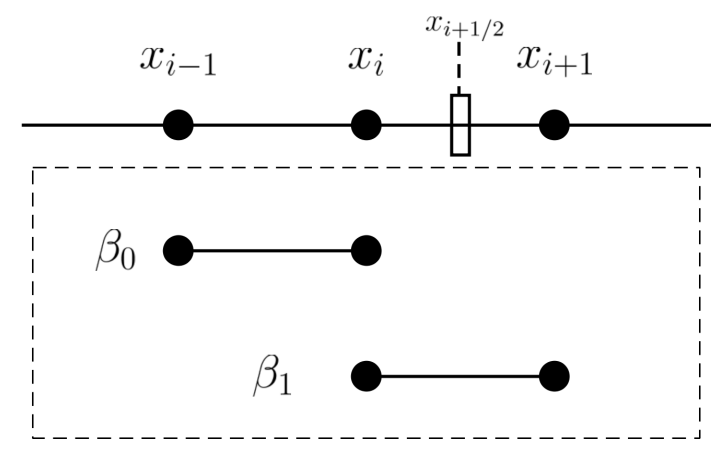

Figure 1: Schematic of stencils for the third-order nonlinear interpolation.

As shown in Fig. 1, a third-order node-to-midpoint interpolation of flow variables can be obtained by using a linear approximation

$$
u_{i+\frac{1}{2}}=-\frac{1}{8} u_{i-1}+\frac{3}{4} u_{i}+\frac{3}{8} u_{i+1}
$$

on a stencil of 3-point, namely, $S_{i+\frac{1}{2}}=\left\{x_{i-1}, x_{i}, x_{i+1}\right\}$.

This optimal third-order scheme can be equivalently represented by using two second-order polynomials, i.e., $u_{i+\frac{1}{2}, 0}$, and $u_{i+\frac{1}{2}, 1}$, each constructed on the two-point sub-stencil $S_{i+\frac{1}{2}, 0}=\left\{x_{i-1}, x_{i}\right\}$, and $S_{i+\frac{1}{2}, 1}=$ $\left\{x_{i}, x_{i+1}\right\}$, respectively, given by

$$
\begin{aligned}
& u_{i+\frac{1}{2}, 0}=\frac{1}{2}\left(3 u_{i}-u_{i-1}\right), \\
& u_{i+\frac{1}{2}, 1}=\frac{1}{2}\left(u_{i}+u_{i+1}\right) .
\end{aligned}
$$

As mentioned above, combinations of low-order interpolations in Eq. (7) yield an equivalent representation of the optimal scheme

$$
u_{i+\frac{1}{2}}=d_{0} u_{i+\frac{1}{2}, 0}+d_{1} u_{i+\frac{1}{2}, 1} .
$$


where the optimal weights are $d_{0}=1 / 4$, and $d_{1}=3 / 4$.

Nonlinear weights are invoked to replace the optimal weights in order for the scheme to suppress spurious oscillations near discontinuities. For instance, the JS weights of Jiang \& Shu [19] being widely used take the form of

$$
\omega_{k}=\frac{\alpha_{k}}{\alpha_{0}+\alpha_{1}}, \quad \alpha_{k}=\frac{d_{k}}{\left(\beta_{k}+\epsilon\right)^{2}}, \quad k=0,1,
$$

where the small parameter $\epsilon=10^{-6}$ is specified to avoid the denominator becoming zero, and $\beta_{k}$ is the local smoothness indicator of each sub-stencil, which is defined as

$$
\begin{aligned}
& \beta_{0}=\left(u_{i}-u_{i-1}\right)^{2}, \\
& \beta_{1}=\left(u_{i+1}-u_{i}\right)^{2} .
\end{aligned}
$$

\subsection{The present method}

As aforementioned, a smoothness measurement yielding strong scale-separation is necessary before the application of stencil selection procedure. Other than using the original scale-separation function in 25], a more efficient function in the form of JS weight is adopted in this work, which is given by

$$
\gamma_{k}=\frac{1}{\left(\beta_{k}+\varepsilon\right)^{q}}, \quad k=0,1
$$

It has been found that the local smoothness indicator $\beta_{k}$ covers a wide range of values from $\mathcal{O}\left(10^{-15}\right)$ in smooth regions up to $\mathcal{O}(1)$ near singularities. The small threshold value employed for the reported studies was $\epsilon=10^{-12}$, which is consistent with the double precision arithmetic used here. In [28, a value $\epsilon=10^{-40}$ was employed with quadruple precision arithmetic, but other larger values, e.g., $10^{-6}$, were tested. The integer power, $q$, plays a key role for the following scale separation mechanism [23]. Here, as readily suggested in Eq. 11, a large value of $q$ can increase the scale of difference among smooth measurements, thus yielding effective detection of discontinuities. Too large a value of $q$, however, tends to treat those smooth wave-like solutions as discontinuous ones. As a compromise, a value of $q=6$ is particularly selected for the effectiveness of strong scale separation in this work.

Instead of using the original nonlinear smoothness measurement in Eq. [11], $\gamma_{k}$ is further normalized as

$$
\chi_{k}=\frac{\gamma_{k}}{\gamma_{0}+\gamma_{1}}, \quad k=0,1
$$

Then a sharp cut-off function can be defined as

$$
\delta_{k}=\left\{\begin{array}{lc}
0, & \text { if } \quad \chi_{k}<C_{T}, \\
1, & \text { otherwise }
\end{array} \quad k=0,1\right.
$$

where $C_{T}$ serves as the global reference for smoothness indicators, and a constant value $C_{T}=10^{-7}$ is used for the computations in this work. Here, if $\delta_{k}$ equals zero, the polynomial evaluated on the corresponding sub-stencil is deemed to be "oscillatory"; otherwise the sub-stencil is regarded as "smooth". Therefore, this procedure works essentially as a discontinuity-detector. 
Table 1: The coefficients of three possible high-order polynomials for the midpoint spatial interpolation $u_{i+\frac{1}{2}}$.

\begin{tabular}{ccccc}
\hline$\left(\delta_{0}, \delta_{1}\right)$ & $\hat{u}_{i+\frac{1}{2}, m}^{*}$ & $a_{m, i-1}$ & $a_{m, i}$ & $a_{m, i+1}$ \\
\hline$(1,1)$ & $u_{i+\frac{1}{2}, 0}^{*}$ & $-1 / 8$ & $3 / 4$ & $3 / 8$ \\
$(1,0)$ & $u_{i+\frac{1}{2}, 1}^{*}$ & $-1 / 2$ & $3 / 2$ & 0 \\
$(0,1)$ & $u_{i+\frac{1}{2}, 2}^{*}$ & 0 & $1 / 2$ & $1 / 2$ \\
\hline
\end{tabular}

The above cut-off function $\delta_{k}$ is further incorporated into the final weighting process to decide whether the contribution of each candidate stencil is taken into account. The resulting weight functions are now given by

$$
\omega_{k}=\frac{d_{k} \delta_{k}}{d_{0} \delta_{0}+d_{1} \delta_{1}}, \quad k=0,1
$$

where $d_{k}$ denotes the optimal linear weight. Two promising advantages of this weighting procedure can be readily shown. Firstly, by removing the stencil crossing discontinuity completely, the numerical robustness can be ensured. Secondly, the underlying third-order linear scheme is expected to be fully recovered in smooth regions, yielding high resolution results with the optimal accuracy.

In fact, the stencil selection procedure has significantly simplified the nonlinear convex combination of candidate stencils. The JS weights in Eq. (9) are in the form of continuous varying functions, which lead into infinite possible combinations for the midpoint interpolation. Only three possible high-order polynomials, however, are being "selected" by the present method since the contribution of each candidate stencil is narrowed down to two possible results, i.e., $d_{k}$ or 0 , corresponding to the stencil "switched on/off", respectively. It should be noted that the above stencil selection procedure assures that at least one of the candidate stencils is used for the interpolation.

The resulting three possible high-order polynomials can be represented in a generic form

$$
u_{i+\frac{1}{2}, m}^{*}=a_{m, i-1} u_{i-1}+a_{m, i} u_{i}+a_{m, i+1} u_{i+1}, \quad m=0,1,2,
$$

each along with its coefficients given in Table 1 .

\subsection{Approximate dispersion relation}

Here, the approximate dispersion relation (ADR) analysis introduced by Pirozzoli [29] is performed to evaluate spectral properties of the numerical schemes considered in the present work, viz., WCNS-JS, WCNS-Z using the nonlinear weight of Borges et al. 30, and the proposed method, still denoted as TCNS.

Particularly, ADR analysis shows the dissipation and dispersion errors of nonlinear numerical schemes in wavenumber space by solving a linear advection equation with a smooth initial condition including different Fourier modes supported on a given grid. A reduced (normalized to lie in the interval $[0, \pi]$ ) wavenumber $\xi$ is defined for all supported modes, and its real $\left(\xi_{R}\right)$ and imaginary $\left(\xi_{I}\right)$ parts represent dispersion and dissipation properties, respectively, for the numerical scheme being evaluated. Results are shown in Fig. 2 
Considerable improvements of both dispersion and dissipation properties are obtained by TCNS compared with WCNS-JS and WCNS-Z in intermediate and high-wavenumber regimes.

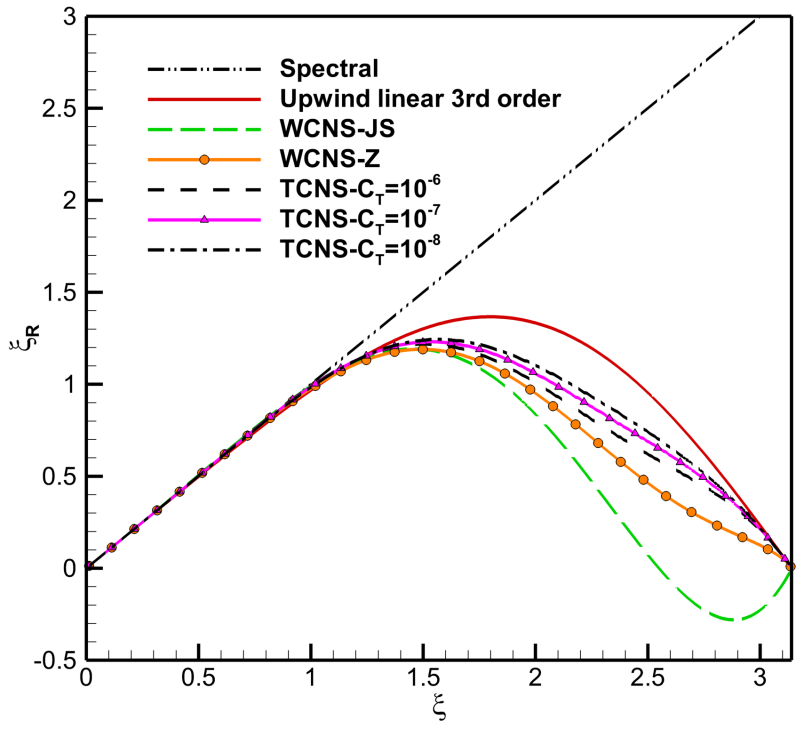

(a)

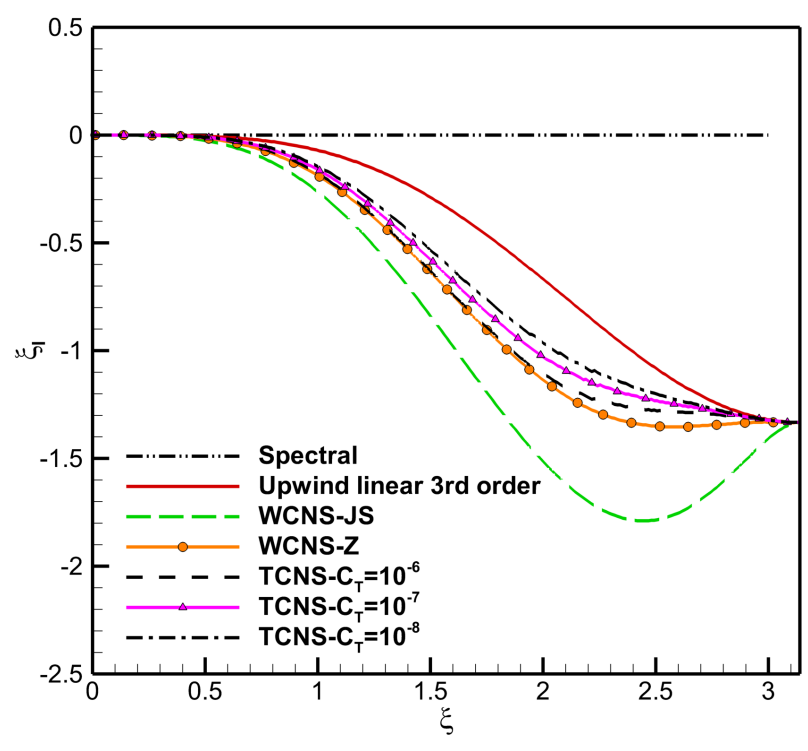

(b)

Figure 2: Approximate dispersion and dissipation properties of third-order schemes; (a) dispersion, and (b) dissipation.

In particular, impact of different threshold parameter $C_{T} \mathrm{~s}$ is investigated here. Comparisons among the results of TCNS using variant $C_{T} \mathrm{~s}$ indicate that TCNS delays the separation from the underlying linear scheme by gradually decreasing $C_{T}$. It can also be inferred by Eq. 13 that the underlying linear scheme is perfectly recovered as $C_{T}=0$. TCNS with small threshold parameter $C_{T}$ is preferred for resolving high-frequency fluctuations, but not for suppressing spurious oscillations near discontinuities. $C_{T}=10^{-7}$ is therefore used as a compromise of both robust shock-capturing capability, and high resolutions for small scales.

\section{Numerical results}

A variety of canonical problems are simulated to assess performance of the present method, compared against WCNS-JS and WCNS-Z. The one-dimensional linear advection equation, and the Euler equations of gas dynamics are used as model equations in this section. Node-to-midpoint interpolation is performed on characteristic fields to alleviate spurious oscillations [1, and the HLL scheme [31] is employed for computation of fluxes, except that in subsection 3.6. a more accurate flux function, the HLLC scheme [32] is also used. Time integration is performed by using the third-order explicit Strong Stability Preserving (SSP) RungeKutta method 27. We note that a CFL number equal to 0.5 has been used in computing test cases reported herein, except that in the linear advection problem of subsection 3.1 the CFL numbers are sufficiently small to achieve temporally stable and converged results. 
The one-dimensional Gaussian pulse advection problem [33] is used to assess the order of accuracy of the proposed schemes when solutions are smooth. This problem is modeled by the linear advection equation

$$
\frac{\partial u}{\partial t}+\frac{\partial u}{\partial x}=0, \quad x \in[0,1],
$$

with periodic boundary conditions and initial condition

$$
u(x, 0)=e^{-300\left(x-x_{c}\right)^{2}}, \quad x_{c}=0.5 .
$$

Temporal integration is performed up to $t=1$, corresponding to one period of single wave propagation in time. Uniformly-spaced grids are progressively refined by a factor of two from the coarsest grid of $N=51$ points. As noted above, the numerical simulation on each grid is conducted using sufficiently small time steps so as to achieve temporally converged results.

Table 2 and Table 3 illustrate numerical errors in terms of $L^{1}$ norm and $L^{\infty}$ norm, respectively, and their convergence rates of the various numerical schemes used. Norm errors and convergence rates of the numerical schemes used are also shown in Fig. 3 Accuracy of all nonlinear schemes, such as WCNS-JS, WCNS-Z and TCNS, fails to coincide with that of the third-order linear scheme, indicating that the optimal linear scheme is not recovered in this case. TCNS obtains relatively higher order of accuracy and higher resolution than WCNS-Z. Despite that the WCNS-JS exhibits higher order of accuracy than the WCNS-Z and TCNS as the mesh is refined from 801 to 1601, comparison of their errors indicates that WCNS-JS has lower resolution than the other two schemes. The ostensible high order of accuracy (higher than 3) obtained by WCNS-JS is caused by the small parameter $\epsilon=10^{-6}$. The interested reader can refer to the work of Henrick et al. 28, for more details. The TCNS obtains improved resolution for the focused smooth field in this case, as compared against two WCNSs used.

Table 2: $\quad L^{1}$-error and convergence rate of different third-order schemes solving the $1 \mathrm{D}$ linear advection equation at $t=1$.

\begin{tabular}{ccccccccc}
\hline \multirow{2}{*}{ DOFs s } & \multicolumn{2}{c}{ Linear } & \multicolumn{2}{c}{ WCNS-JS } & \multicolumn{2}{c}{ WCNS-Z } & \multicolumn{2}{c}{ TCNS } \\
\cline { 2 - 9 } & Error & Order & Error & Order & Error & Order & Error & Order \\
\hline 51 & $2.91 \mathrm{E}-2$ & $*$ & $6.60 \mathrm{E}-2$ & $*$ & $5.07 \mathrm{E}-2$ & $*$ & $4.35 \mathrm{E}-2$ & $*$ \\
101 & $6.84 \mathrm{E}-3$ & 2.09 & $2.99 \mathrm{E}-2$ & 1.14 & $1.80 \mathrm{E}-2$ & 1.5 & $1.62 \mathrm{E}-2$ & 1.42 \\
201 & $1.03 \mathrm{E}-3$ & 2.73 & $9.17 \mathrm{E}-3$ & 1.71 & $4.77 \mathrm{E}-3$ & 1.91 & $3.41 \mathrm{E}-3$ & 2.25 \\
401 & $1.33 \mathrm{E}-4$ & 2.95 & $2.47 \mathrm{E}-3$ & 1.9 & $1.14 \mathrm{E}-3$ & 2.07 & $6.44 \mathrm{E}-4$ & 2.41 \\
801 & $1.68 \mathrm{E}-5$ & 2.99 & $4.55 \mathrm{E}-4$ & 2.44 & $2.52 \mathrm{E}-4$ & 2.17 & $1.23 \mathrm{E}-4$ & 2.38 \\
1601 & $2.10 \mathrm{E}-6$ & 3.00 & $5.42 \mathrm{E}-5$ & 3.07 & $5.22 \mathrm{E}-5$ & 2.27 & $2.33 \mathrm{E}-5$ & 2.41 \\
\hline
\end{tabular}


Table 3: $\quad L^{\infty}$-error and convergence rate of different third-order schemes solving the 1D linear advection equation at $t=1$.

\begin{tabular}{ccccccccc}
\hline \multirow{2}{*}{ DOFs } & \multicolumn{2}{c}{ Linear } & \multicolumn{2}{c}{ WCNS-JS } & \multicolumn{2}{c}{ WCNS-Z } & \multicolumn{2}{c}{ TCNS } \\
\cline { 2 - 9 } & Error & Order & Error & Order & Error & Order & Error & Order \\
\hline 51 & $2.20 \mathrm{E}-1$ & $*$ & $5.27 \mathrm{E}-1$ & $*$ & $4.46 \mathrm{E}-1$ & $*$ & $4.12 \mathrm{E}-1$ & $*$ \\
101 & $6.27 \mathrm{E}-2$ & 1.82 & $3.14 \mathrm{E}-1$ & 0.75 & $2.26 \mathrm{E}-1$ & 0.98 & $2.00 \mathrm{E}-1$ & 1.04 \\
201 & $1.05 \mathrm{E}-2$ & 2.58 & $1.41 \mathrm{E}-1$ & 1.15 & $9.37 \mathrm{E}-2$ & 1.27 & $7.30 \mathrm{E}-2$ & 1.45 \\
401 & $1.39 \mathrm{E}-3$ & 2.91 & $5.40 \mathrm{E}-2$ & 1.39 & $3.52 \mathrm{E}-2$ & 1.41 & $2.42 \mathrm{E}-2$ & 1.59 \\
801 & $1.75 \mathrm{E}-4$ & 2.99 & $1.64 \mathrm{E}-2$ & 1.72 & $1.26 \mathrm{E}-2$ & 1.48 & $7.77 \mathrm{E}-3$ & 1.64 \\
1601 & $2.20 \mathrm{E}-5$ & 3.00 & $3.28 \mathrm{E}-3$ & 2.32 & $4.43 \mathrm{E}-3$ & 1.51 & $2.58 \mathrm{E}-3$ & 1.59 \\
\hline
\end{tabular}

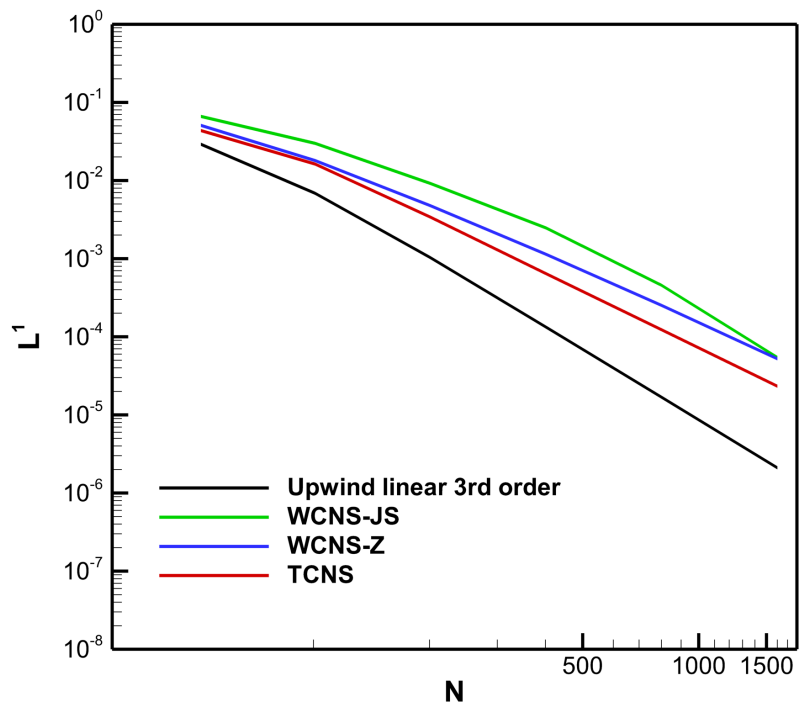

(a) $L^{1}$ error

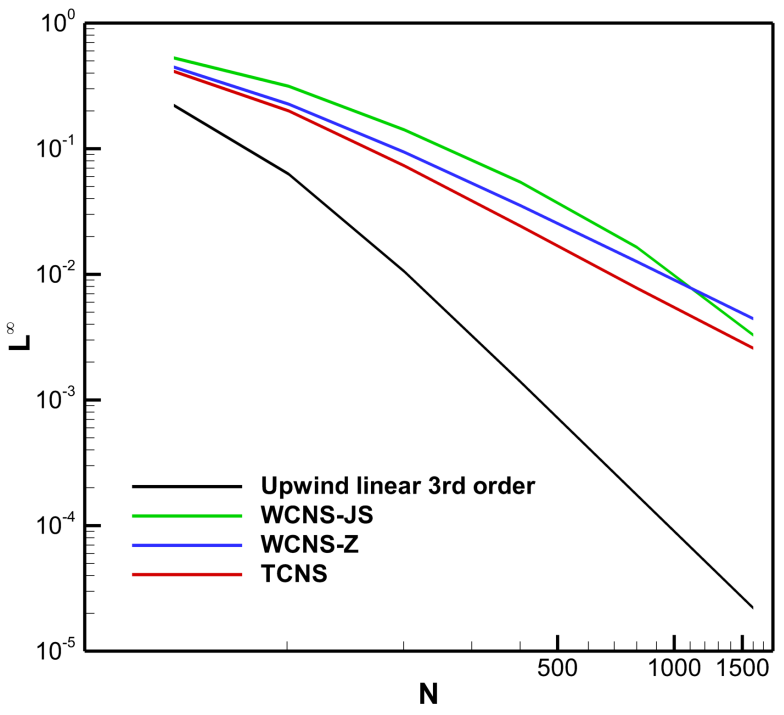

(b) $L^{\infty}$ error

Figure 3: Spatial convergence rate of third-order schemes for $L^{1}$ - and $L^{\infty}$-errors in solutions to the 1D linear advection equation at $t=1$.

\subsection{Sod and Lax shock tube problems}

Riemann initial-value problems of Sod [34] and Lax [35] are used to further evaluate shock-capturing capability of the proposed schemes employing discretization of the 1-D Euler equations

$$
\begin{gathered}
\frac{\partial \rho}{\partial t}+\frac{\partial(\rho u)}{\partial x}=0 \\
\frac{\partial(\rho u)}{\partial t}+\frac{\partial\left(\rho u^{2}\right)}{\partial x}=-\frac{\partial p}{\partial x} \\
\frac{\partial E}{\partial t}+\frac{\partial(u E)}{\partial x}=-\frac{\partial(u p)}{\partial x},
\end{gathered}
$$

where $E=e+\frac{1}{2} u^{2}$ is the total energy per unit mass, and $e$ is internal energy. The dependent variables are 
equations system.

The Sod shock tube problem involves a right-moving shock of Mach number 1.7, while for the Lax shock tube problem, the right-moving shock has Mach number 2.0. Initial conditions for the Sod problem are

$$
(\rho, u, p)=\left\{\begin{array}{cc}
(1,0,1) & x \in[0,0.5] \\
(0.125,0,0.1) & x \in(0.5,1]
\end{array}\right.
$$

and the results at $t=0.2$ are given by solving the problem on an evenly-distributed grid of $N=101$ points.

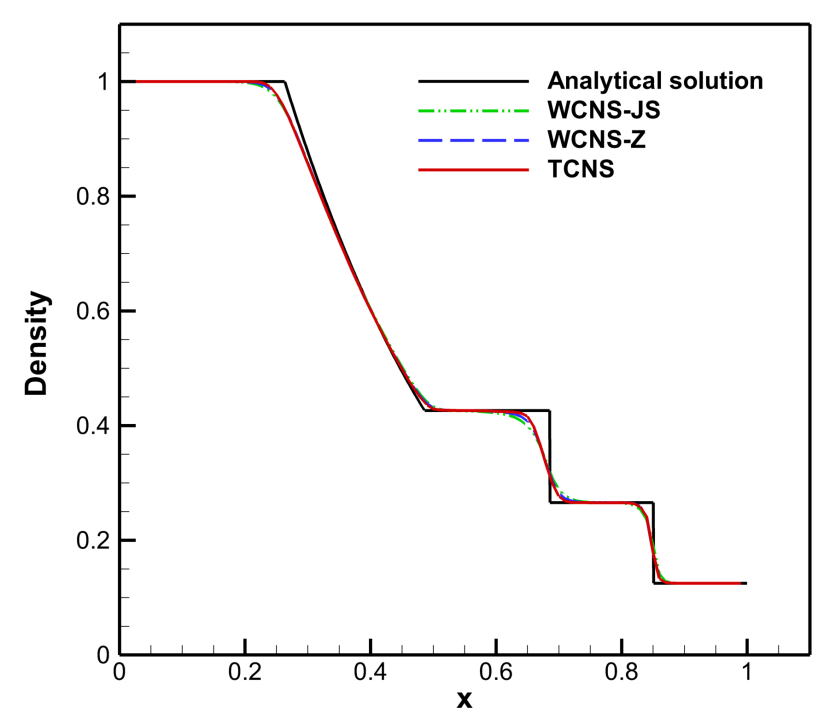

(a)

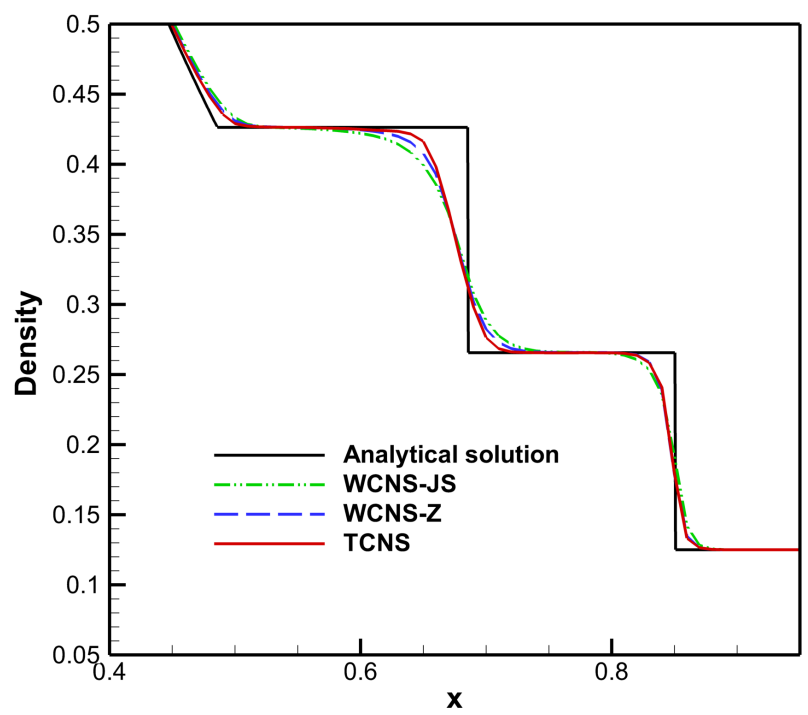

(b)

Figure 4: Numerical and exact solutions for Sod problem at $t=0.2$; (a) density, (b) zoom in of density.

Density distributions are better resolved using TCNS than WCNS-JS and WCNS-Z as shown in Fig. 4 The results are free of spurious oscillations near discontinuities, showing the decent shock-capturing capability of these nonlinear schemes.

Initial conditions for the Lax shock-tube problem are

$$
(\rho, u, p)=\left\{\begin{array}{cc}
(0.445,0.698,3.528) & x \in[0,0.5] \\
(0.5,0,0.571) & x \in(0.5,1] .
\end{array}\right.
$$

This case is also simulated on an evenly distributed grid of $N=101$ points, and the results at $t=0.14$ are shown in Fig. 5 for density and velocity distributions.

Overall, the solutions of all nonlinear schemes are reasonably decent. The WCNS-JS smears the contact discontinuity, indicating too much numerical dissipation. Sharp profiles are obtained by the TCNS over two WCNSs near discontinuities. Minor overshoot is noticeable for the velocity profiles of all schemes used near the expansion fan; but such overshoots are considered to be acceptable since similar behaviors are also seen 


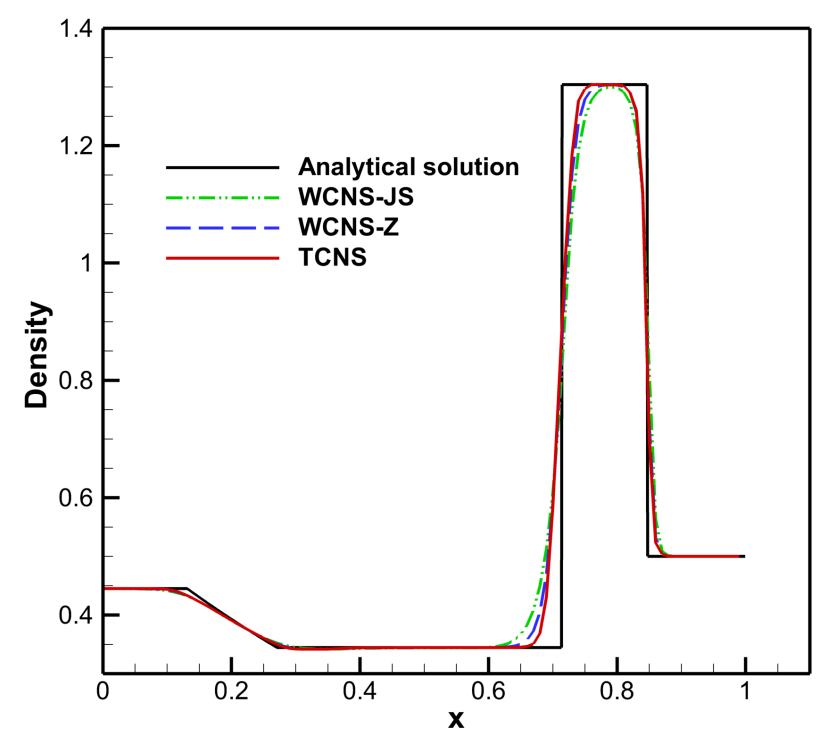

(a)

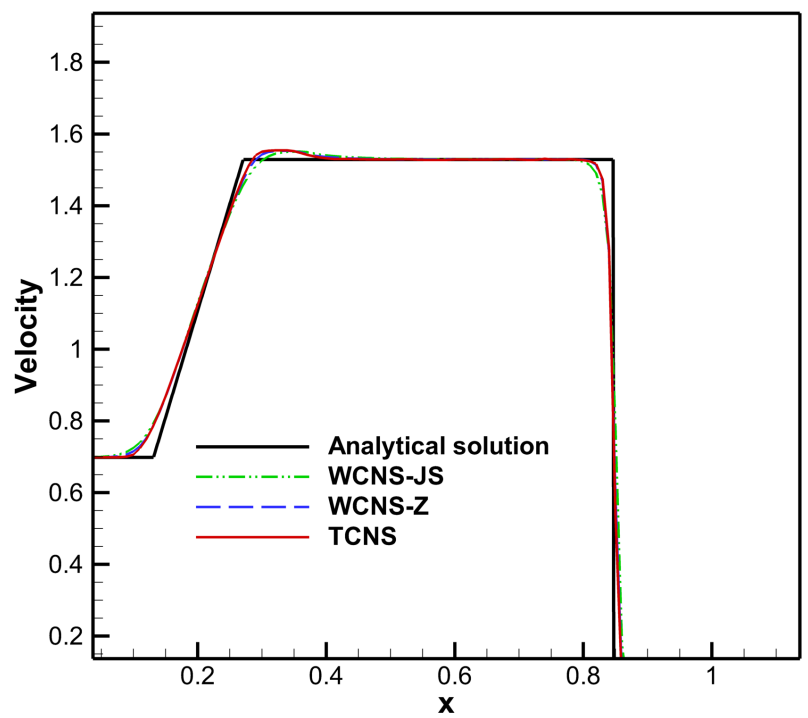

(b)

Figure 5: Lax problem numerical and exact solutions at $t=0.14$; (a) density, and (b) velocity.

in various other high-order schemes and simply reflect "essentially," rather than completely (e.g., TVD), non-oscillatory behavior.

\subsection{Shock/density-wave interaction of Osher-Shu}

The shock/density-wave interaction problem of Osher and Shu 33 is characterized by a right-moving shock wave of Mach number 3 interacting with high-frequency sine waves in the density field. A multi-scale wave structure evolves after the shock wave interacts with the oscillating density wave, and both shockcapturing and wave-resolution capabilities are evaluated for the methods considered herein via this problem.

The problem again employs the 1-D Euler equations and is initialized by

$$
(\rho, u, p)= \begin{cases}(3.857,2.629,10.333), & x \in[0,1], \\ (1+0.2 \sin (5 x), 0,1), & x \in(1,10] .\end{cases}
$$

This case is run on a grid of $N=801$ points which are uniformly distributed, and the final time is $t=1.8$.

Since there is no theoretical solution for this problem, a fine-grid numerical solution via the WCNS-JS scheme on a grid of $N=4001$ points is used as a reference.

As shown in Fig. 6. TCNS and WCNS-Z produce considerably better resolved density waves behind the shock wave compared with WCNS-JS. Minor improvement is exhibited by the TCNS over the WCNS-Z, which is expected because of its lower dissipation property in intermediate and high-wavenumber regimes in spectral space. 


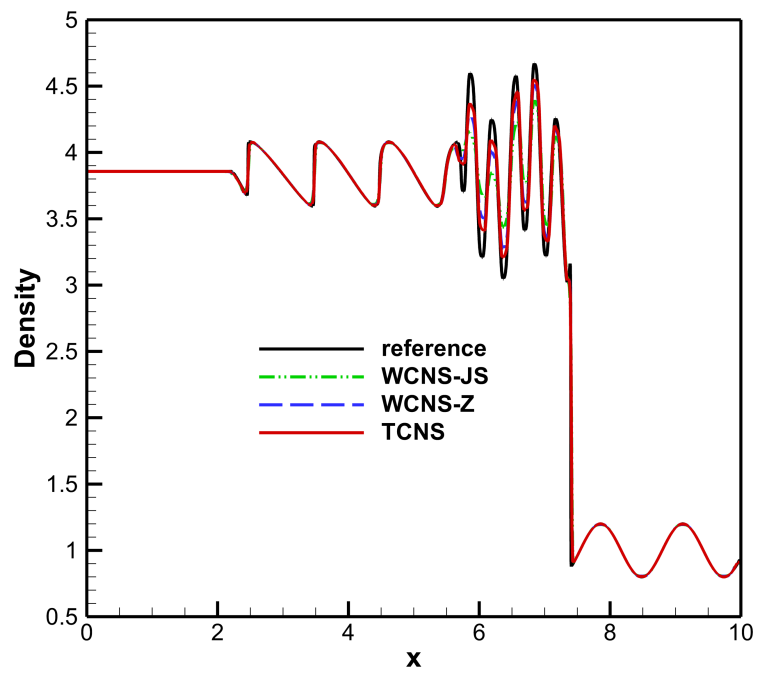

(a)

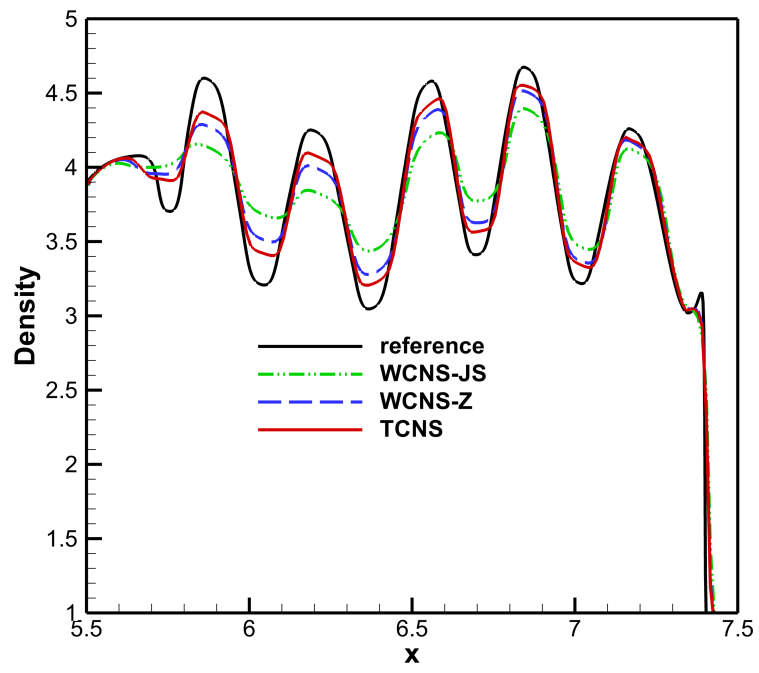

(b)

Figure 6: Shock/density-wave interaction problem; numerical solutions and the exact solution at $t=1.8$; (a) full spatial domain, and (b) zoom in on high-amplitude region.

\subsection{Two-dimensional Riemann problems}

In this section, the configurations 3 and 6 out of 19 2-D Riemann problems used by Lax and Liu [36. are employed to evaluate performance of the currently investigated numerical schemes. In particular, these two configurations are characterized by rich small scales in the flow fields, and thus are specifically chosen to evaluate ability of the proposed numerical schemes to resolve fine flow structures. These 2-D Riemann problems are widely-solved canonical problems in the present context, analogous to the 1-D Sod shock tube problem of the previous section. The equations of motion are the 2-D Euler equations which are a straightforward extension of the 1-D case given above:

$$
\begin{aligned}
\frac{\partial \rho}{\partial t}+\frac{\partial(\rho u)}{\partial x}+\frac{\partial(\rho v)}{\partial y} & =0 \\
\frac{\partial(\rho u)}{\partial t}+\frac{\partial\left(\rho u^{2}\right)}{\partial x}+\frac{\partial(\rho u v)}{\partial y} & =-\frac{\partial p}{\partial x}, \\
\frac{\partial(\rho v)}{\partial t}+\frac{\partial(\rho u v)}{\partial x}+\frac{\partial\left(\rho v^{2}\right)}{\partial y} & =-\frac{\partial p}{\partial y}, \\
\frac{\partial E}{\partial t}+\frac{\partial(u E)}{\partial x}+\frac{\partial(v E)}{\partial y} & =-\frac{\partial(u p)}{\partial x}-\frac{\partial(v p)}{\partial y} .
\end{aligned}
$$




\subsubsection{Configuration 3}

Initial conditions for configuration 3 are given by

$$
(\rho, u, v, p)=\left\{\begin{array}{cl}
(1.5,0,0,1.5) & (x, y) \in\left[\frac{1}{2}, 1\right] \times\left[\frac{1}{2}, 1\right], \\
(0.5323,1.206,0,0.3) & (x, y) \in\left[0, \frac{1}{2}\right] \times\left[\frac{1}{2}, 1\right], \\
(0.138,1.206,1.206,0.029) & (x, y) \in\left[0, \frac{1}{2}\right] \times\left[0, \frac{1}{2}\right], \\
(0.5323,0,1.206,0.3) & (x, y) \in\left[\frac{1}{2}, 1\right] \times\left[0, \frac{1}{2}\right] .
\end{array}\right.
$$

Boundary conditions are

$$
\begin{array}{ll}
\frac{\partial \mathbf{u}(x, y, t)}{\partial x}=0, & x=0,1, \quad \forall y, t, \\
\frac{\partial \mathbf{u}(x, y, t)}{\partial y}=0, & y=0,1, \quad \forall x, t,
\end{array}
$$

where the vector of primitive variables is given by $\mathbf{u}=(\rho, u, v, p)^{T}$. Image nodes are used adjacent to each boundary of the domain to implement these conditions. It should be noted that computations were terminated before the shock structures interacted with boundaries; thus the discussion will be focused on the central part of the flow field.

Figure 7 presents results obtained with $2049^{2}$ grid points with uniform spacing using all of WCNS-JS, WCNS-Z, and TCNS, at $t=0.3$. The TCNS captures more vortices along contact lines than do WCNSJS and WCNS-Z. The resolution obtained by the WCNS-Z is slightly higher than that of the WCNS-JS. Without physical results for comparisons, one must question whether this numerically-produced "turbulence" actually represents physics. In fact, Shi et al. 37. suggest that during a mesh refinement slip lines usually are non-physical and depend on numerical scheme being used. However, these simulations are important for identifying the magnitude of numerical viscosities. Schemes with low dissipations are expected to capture rich small scales in this specific flow region; such is the case for the TCNS.

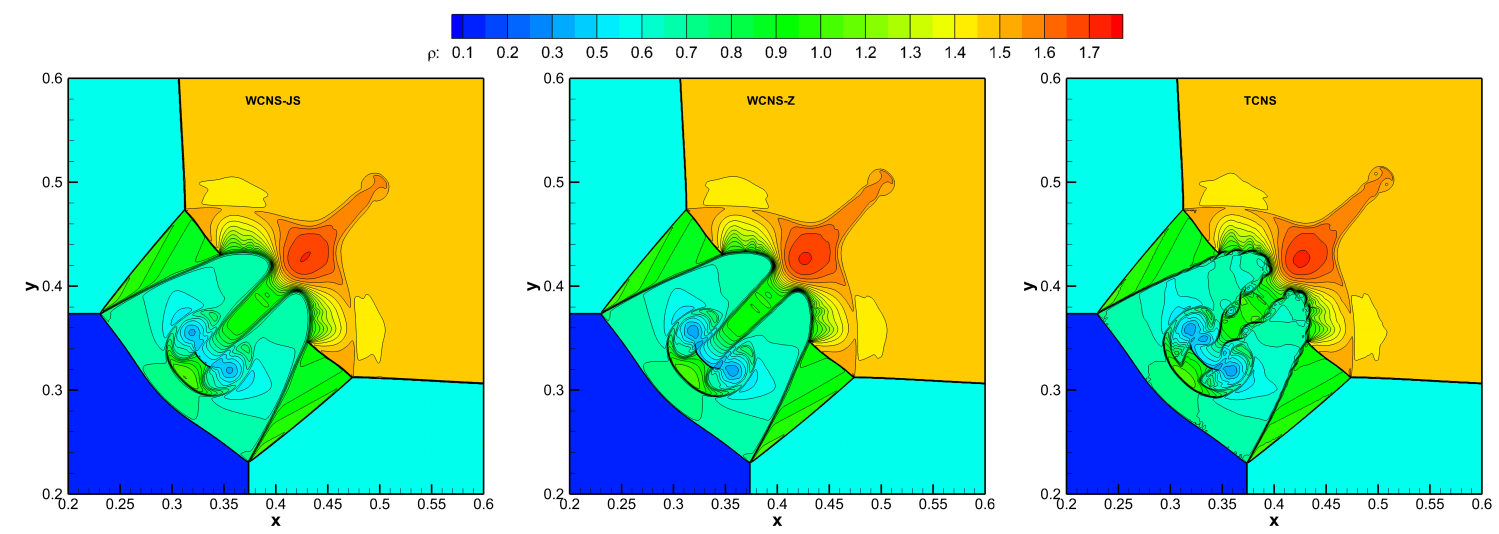

Figure 7: Configuration 3 of 2-D Riemann problems in [36; 30 density contour lines ranging from 0.1 to 1.8 at $t=0.3$. 


\subsubsection{Configuration 6}

The equations of motion are the same as those used for configuration 3, the 2-D Euler equations, but now with initial conditions given by

$$
(\rho, u, v, p)=\left\{\begin{array}{cc}
(1,0.75,-0.5,1) & (x, y) \in\left[\frac{1}{2}, 1\right] \times\left[\frac{1}{2}, 1\right], \\
(2,0.75,0.5,1) & (x, y) \in\left[0, \frac{1}{2}\right) \times\left[\frac{1}{2}, 1\right], \\
(1,-0.75,0.5,1) & (x, y) \in\left[0, \frac{1}{2}\right) \times\left[0, \frac{1}{2}\right), \\
(3,-0.75,-0.5,1) & (x, y) \in\left[\frac{1}{2}, 1\right] \times\left[0, \frac{1}{2}\right) .
\end{array}\right.
$$

Boundary conditions are the same as in the preceding test case.

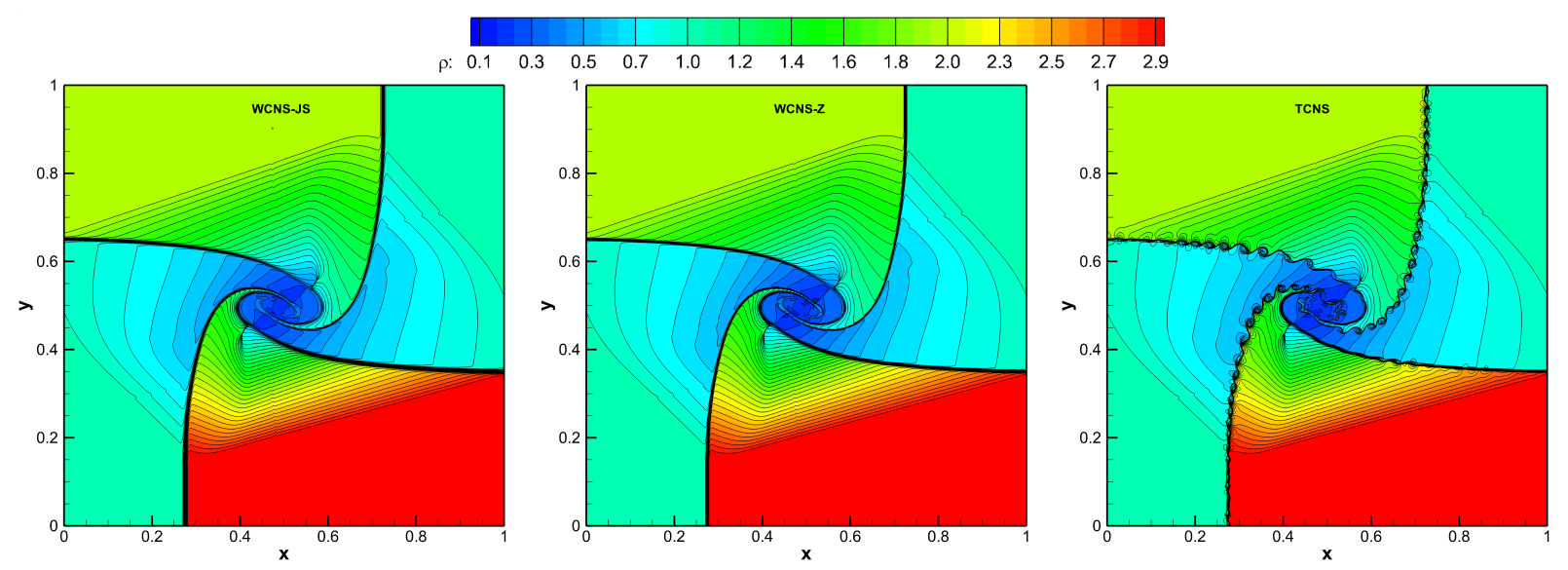

Figure 8: Configuration 6 of 2-D Riemann problems in [36] computed on a grid of $2049 \times 2049$ points; 40 density contour lines ranging from 0.1 to 2.9 at $t=0.3$.

Results obtained on a grid of $2049^{2}$ points with uniform spacing by using the WCNS-JS, WCNS-Z, and TCNS at $t=0.3$ are shown in Fig. 8. The flow structures predicted by two WCNSs are close. However, abundant small-scale flow structures are resolved by the TCNS along contact lines. Similar to the discussion as in the preceding test case, low numerical dissipation of the present scheme is again shown in this case.

\subsection{Double Mach reflection of a strong shock}

A 2-D simulation of a right-moving Mach 10 shock wave over a $30^{\circ}$ wedge is also performed. This specific case was initially investigated by Woodward and Colella [38 to evaluate numerical methods when simulating complex flows structures evolved in time such as the double Mach reflection, the primary and secondary triple points, and the Mach stem etc. It is a demanding test case, therefore is selected for examining performance of the TCNS in both strong discontinuity capturing and complex flow structure resolution.

The 2-D Euler equations are again used as the governing equations. To simply the numerical simulation, the coordinate system is aligned with the wedge. A 2-D domain $[0,4] \times[0,1]$ of uniform Cartesian grids 
$961 \times 241$ is used for the computation. Initial conditions are given by

$$
(\rho, u, v, p)=\left\{\begin{array}{cc}
(1.4,0,0,1) & x \in\left(\frac{1}{6}+\frac{y}{\tan \frac{\pi}{3}}, 4\right] \\
\left(8,8.25 \cos \frac{\pi}{6},-8.25 \sin \frac{\pi}{6}, 116.5\right) & \text { otherwise. }
\end{array}\right.
$$

The inflow boundary condition specified with the post-shock condition is imposed at the left-side of the domain, and the right-side of the domain is set as the generic outflow. At the bottom, the post-shock condition is imposed in $x \in[0,1 / 6)$, whereas a reflecting wall condition is used for the rest of interval. At the top side of the domain, the boundary condition is specified to describe the exact motion of the incident shock [38].

Temporal simulation is performed up to time $t=0.2$. It is worth to note that all schemes, i.e., WCNS-JS, WCNS-Z and TCNS are capable of stable computation for this severe case. Pressure and density are positive preserved, and there is no need for any corrections. Results of density contours at $t=0.2$ for the full spatial domain are shown in Fig. 9 and for the zoomed-in region near the triple point in Fig. 10, respectively. It can be clearly observed that all schemes capture the strong shocks and the Mach stem without oscillations. The TCNS has higher resolution for the second reflected shock, and the primary slip line than two WCNSs. The small vortices rolling up alone the slip line is also better resolved by the TCNS; the WCNS-JS yields the lowest resolution, and the WCNS-Z lies between.
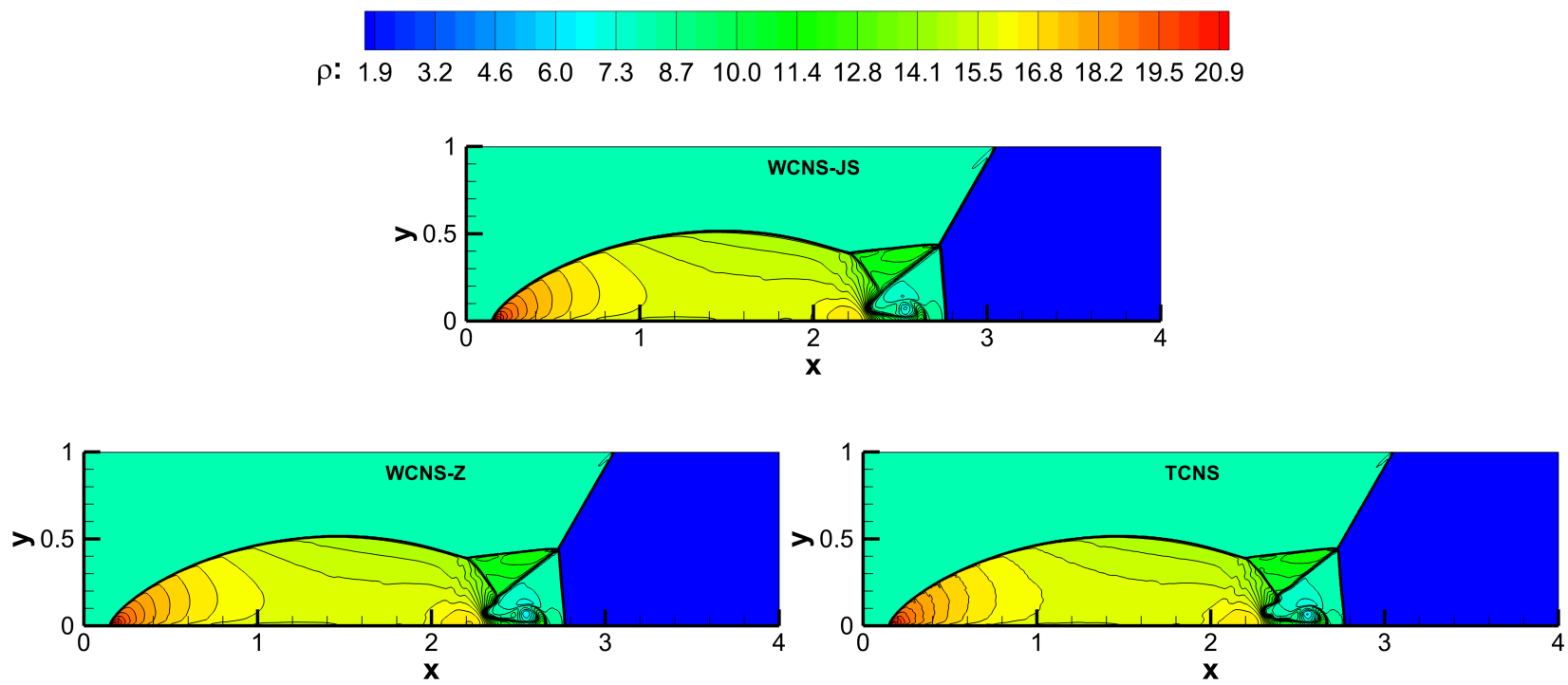

Figure 9: Double Mach reflection of a strong shock in 38 computed on a grid of $961 \times 241$ points; 43 density contour lines ranging from 1.887 to 20.9 at $t=0.2$; full spatial domain.

\subsection{Rayleigh-Taylor instability}

The Rayleigh-Taylor instability problem, which contains both discontinuities and complex flow structures, has been widely studied, both experimentally and computationally; and it is used here as a final example 

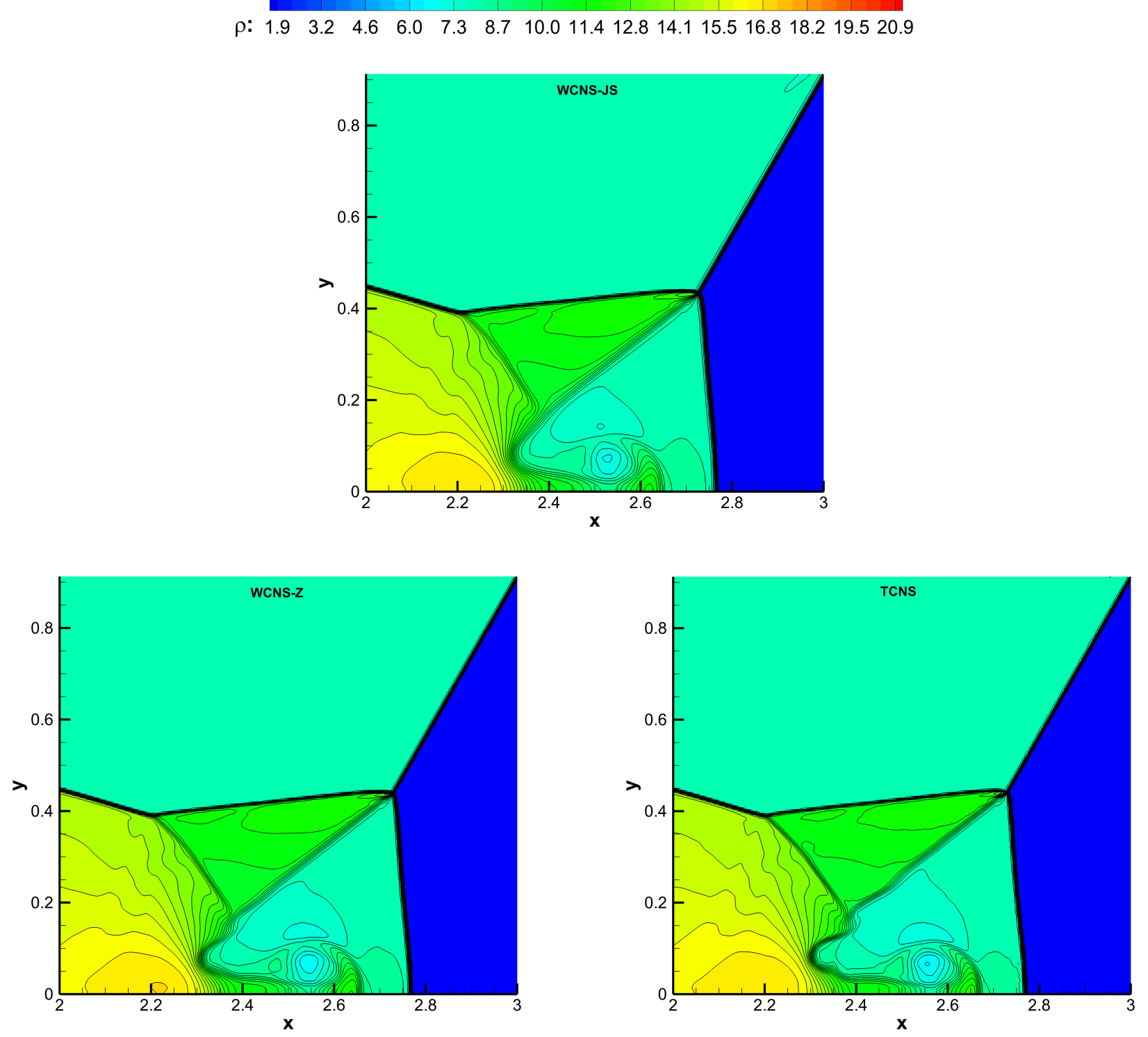

Figure 10: Double Mach reflection of a strong shock in [38]computed on a grid of $961 \times 241$ points; 43 density contour lines ranging from 1.887 to 20.9 at $t=0.2$; zoom in on the region around the triple point.

for examining performance of the presently-studied methods. Again, the 2-D Euler equations comprise the basic mathematical model. In the present case, initial conditions are given by

$$
(\rho, u, v, p)=\left\{\begin{array}{cl}
(2,0,-0.025 a \cos (8 \pi x), 1+2 y) & (x, y) \in[0,0.25] \times[0,0.5) \\
(1,0,-0.025 a \cos (8 \pi x), y+3 / 2) & (x, y) \in[0,0.25] \times[0.5,1]
\end{array}\right.
$$

where $a$ is the speed of sound, given by $a=\sqrt{\gamma p / \rho}$ and a different $\gamma=\frac{5}{3}$ is used for this specific case. Reflecting boundary conditions are imposed at the left- and right-hand sides of the domain; i.e., velocity $u$ adjacent to boundary nodes is assigned the value of corresponding image nodes with sign reversed, and $\rho, v$, 
and $p$ are straightforwardly assigned. Constant boundary conditions are given for the top and bottom sides, expressed as

$$
(\rho, u, v, p)=\left\{\begin{array}{ccc}
(1,0,0,2.5) & y=1, & \forall t, x, \\
(2,0,0,1) & y=0, & \forall t, x .
\end{array}\right.
$$

Two source terms, $\rho$ and $\rho v$, are added to the right-hand side of the third and the fourth equations, respectively, of the 2-D Euler system given at the beginning of this subsection, as done by Shi et al. [37, among

\section{Acknowledgments}

This work is partly supported by the National Numerical Wind Tunnel Project (Grant No. NNW2018ZT4B09) and the National Natural Science Foundation of China (Grant No. 11872144). 


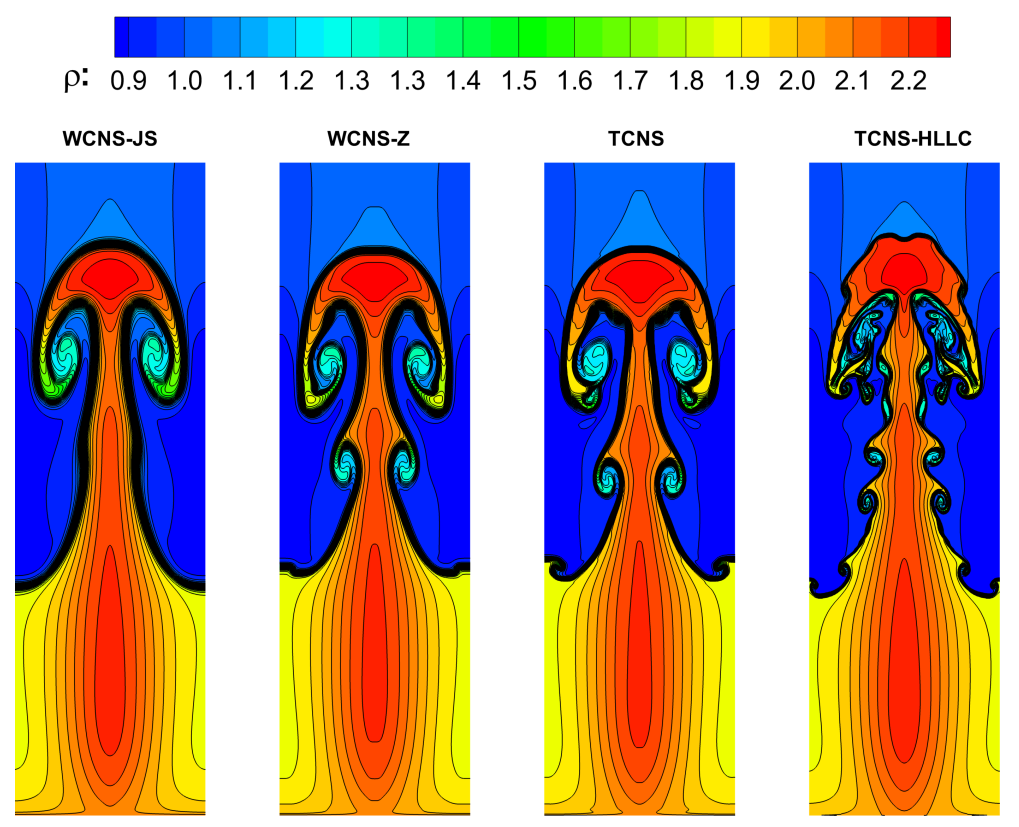

(a) Grid resolution $257 \times 1025$
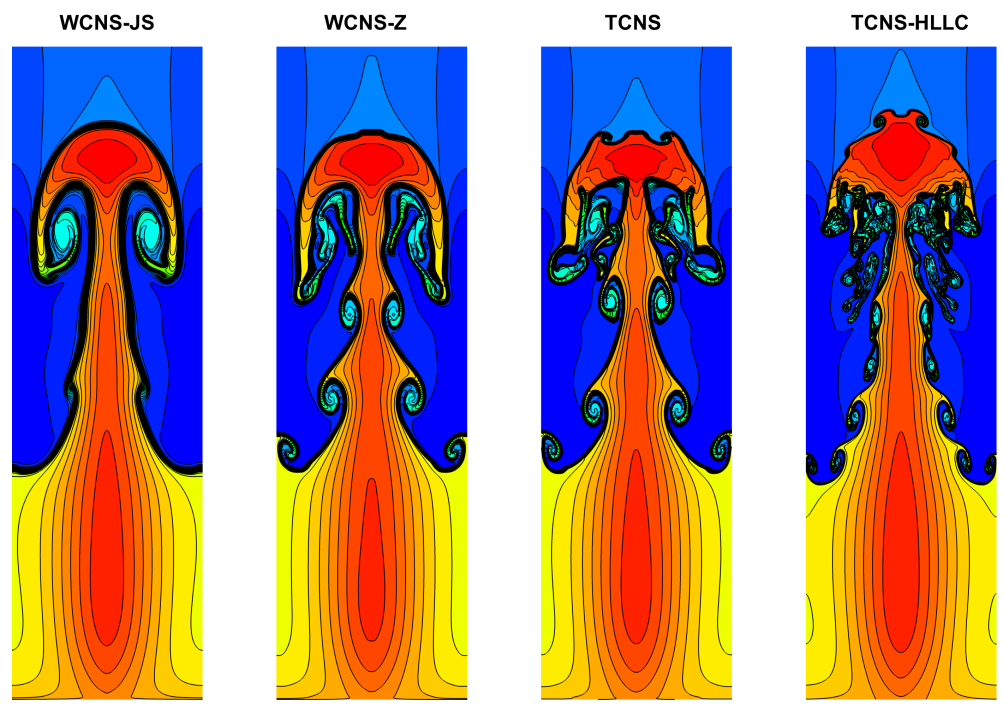

(b) Grid resolution $513 \times 2049$

Figure 11: Rayleigh-Taylor instability problem; 30 density contour lines ranging from 0.9 to 2.2 at $t=1.95$.

\section{References}

[1] X. Deng, H. Zhang, Developing high-order weighted compact nonlinear schemes, Journal of Computational Physics 165 (1) (2000) 22 - 44. doi:10.1006/jcph.2000.6594.

[2] Z. Wang, K. Fidkowski, R. Abgrall, F. Bassi, D. Caraeni, A. Cary, H. Deconinck, R. Hartmann, K. Hillewaert, H. Huynh, N. Kroll, G. May, P.-O. Persson, B. van Leer, M. Visbal, High-order cfd methods: current status and perspective, Int. J. Numer. Meth. Fluids 72 (8) (2013) 811-845. 
[3] E. F. Toro, Riemann Solvers and Numerical Methods for Fluid Dynamics: A Practical Introduction, 3rd Edition, Springer-Verlag Berlin Heidelberg, 2009.

[4] S. Pirozzoli, Conservative hybrid compact-weno schemes for shock-turbulence interaction, Journal of Computational Physics 178 (1) (2002) 81-117. doi:10.1006/jcph.2002.7021.

[15] X. D. Liu, S. Osher, T. Chan, Weighted essentially non-oscillatory schemes, Journal of Computational Physics 115 (1) (1994) 200-212.

[5] A. Harten, High resolution schemes for hyperbolic conservation laws, Journal of Computational Physics 49 (3) (1983) 357-393.

[6] S. K. Lele, Compact finite difference schemes with spectral-like resolution, Journal of Computational Physics 103 (1) (1992) 16 - 42. doi:10.1016/0021-9991(92)90324-R.

[7] P. Tsoutsanis, A. F. Antoniadis, D. Drikakis, Weno schemes on arbitrary unstructured meshes for laminar, transitional and turbulent flows, Journal of Computational Physics 256 (2014) $254-276$. doi:https://doi.org/10.1016/j.jcp.2013.09.002

[8] P. Tsoutsanis, A. F. Antoniadis, K. W. Jenkins, Improvement of the computational performance of a parallel unstructured weno finite volume cfd code for implicit large eddy simulation, Computers \& Fluids 173 (2018) 157 - 170. doi:https://doi.org/10.1016/j.compfluid.2018.03.012.

[9] X. Deng, H. Maekawa, Compact high-order accurate nonlinear schemes, Journal of Computational Physics 130 (1) (1997) 77 - 91. doi:10.1006/jcph.1996.5553.

[10] T. Nonomura, N. Iizuka, K. Fujii, Freestream and vortex preservation properties of high-order weno and (1. wens on curvilinear grids, Computers \& Fluids 39 (2) (2010) 197 - 214. doi:10.1016/j.compfluid. 2009.08 .005

[11] T. Nonomura, K. Fujii, Robust explicit formulation of weighted compact nonlinear scheme, Computers \& Fluids 85 (2013) 8 - 18, international Workshop on Future of CFD and Aerospace Sciences. doi: 10.1016/j.compfluid.2012.09.001.

[12] X. Deng, High-order accurate dissipative weighted compact nonlinear schemes, Science in China Series A: Mathematics 45 (3) (2002) 356. doi:10.1360/02ys9037.

[13] X. Deng, M. Mao, Y. Jiang, H. Liu, New high-order hybrid cell-edge and cell-node weighted compact nonlinear schemes, in: 20th AIAA Computational Fluid Dynamics Conference, AIAA Paper 2011-3857, Honolulu, Hawaii, 2011. doi:10.2514/6.2011-3857.

[14] M. L. Wong, S. K. Lele, High-order localized dissipation weighted compact nonlinear scheme for shockand interface-capturing in compressible flows, Journal of Computational Physics 339 (2017) 179 - 209. doi:10.1016/j.jcp.2017.03.008. 
[16] P. L. Roe, Approximate Riemann solvers, parameter vectors, and difference schemes, Journal of Computational Physics 43 (2) (1981) 357-372.

[26] H. Zhang, F. Zhang, J. Liu, J. McDonough, C. Xu, A simple extended compact nonlinear scheme with adaptive dissipation control, Communications in Nonlinear Science and Numerical Simulation 84 (2020) 105191. doi:10.1016/j.cnsns.2020.105191.

[27] S. Gottlieb, C. Shu, E. Tadmor, Strong stability-preserving high-order time discretization methods, SIAM Review 43 (1) (2001) 89-112. doi:10.1137/S003614450036757X.

[28] A. K. Henrick, T. D. Aslam, J. M. Powers, Mapped weighted essentially non-oscillatory schemes: Achieving optimal order near critical points, Journal of Computational Physics 207 (2) (2005) 542-567. 
[29] S. Pirozzoli, On the spectral properties of shock-capturing schemes, Journal of Computational Physics 219 (2) (2006) 489 - 497. doi:10.1016/j.jcp.2006.07.009.

[30] R. Borges, M. Carmona, B. Costa, W. S. Don, An improved weighted essentially non-oscillatory scheme for hyperbolic conservation laws, Journal of Computational Physics 227 (6) (2008) 3191-3211. doi: $10.1016 / j \cdot j c p .2007 .11 .038$

[31] B. Einfeldt, On Godunov-type methods for gas dynamics, SIAM Journal on Numerical Analysis 25 (2) (1988) 294-318.

[32] E. Toro, M. Spruce, W. Speares, Restoration of the contact surface in the HLL-Riemann solver, Shock Waves 4 (1) (1994) 25-34.

[33] C.-W. Shu, S. Osher, Efficient implementation of essentially non-oscillatory shock-capturing schemes,ii, J. Comput. Phys. 83 (1) (1989) 32-78. doi:10.1016/0021-9991(89)90222-2.

[34] G. A. Sod, A survey of several finite difference methods for systems of nonlinear hyperbolic conservation laws, Journal of Computational Physics 27 (1) (1978) 1-31.

[35] P. D. Lax, Weak solutions of nonlinear hyperbolic equations and their numerical computation, Communications on Pure and Applied Mathematics 7 (1) (1954) 159-193. doi:10.1002/cpa.3160070112.

[36] P. Lax, X. Liu, Solution of two-dimensional riemann problems of gas dynamics by positive schemes, SIAM Journal on Scientific Computing 19 (2) (1998) 319-340. doi:10.1137/S1064827595291819.

[37] J. Shi, Y.-T. Zhang, C.-W. Shu, Resolution of high order weno schemes for complicated flow structures, Journal of Computational Physics 186 (2) (2003) 690 - 696. doi:10.1016/S0021-9991(03)00094-9.

[38] P. Woodward, P. Colella, The numerical simulation of two-dimensional fluid flow with strong shocks, Journal of Computational Physics 54 (1) (1984) 115-173.

[39] F. Zhang, J. Liu, B. Chen, W. Zhong, Evaluation of rotated upwind schemes for contact discontinuity and strong shock, Computers \& Fluids 134-135 (2016) 11-22. 